\title{
Three-Dimensional Analysis of Facial Asymmetry of Healthy Hispanic Caucasian Children
}

\author{
Juhun LEE ${ }^{a, b}$, Brian KU ${ }^{a}$, Adriana C. DA SILVEIRA ${ }^{a, c}$, Mia K. MARKEY ${ }^{a, b^{*}}$ \\ ${ }^{a}$ The University of Texas at Austin, Austin (TX), USA; \\ ${ }^{\mathrm{b}}$ The University of Texas MD Anderson Cancer Center, Houston (TX), USA; \\ 'Dell Children's Medical Center of Central Texas, Austin (TX), USA
}

\begin{abstract}
The objective of this study was to conduct a quantitative analysis of facial asymmetry of healthy Hispanic Caucasian children residing in Central Texas, USA. 3D facial surface images of healthy Hispanic Caucasian participants (41 boys, 39 girls) currently residing in Central Texas, USA (aged 7 and 12 years) were obtained using a 3dMDcranial system (3dMD, Atlanta, GA) under an approved IRB protocol. Facial features of each hemiface (left and right) were quantified using 27 anthropometric distances between 22 facial landmarks computed from the 3D facial surface images. Two types of distances were included: 1) Euclidean (2 orbital, 3 nasal, 8 oral, and 6 profile) and 2) Geodesic (1 orbital, 1 nasal, 6 profile) distances. The ratio of each facial feature of the left hemiface to the corresponding facial feature of the right hemiface was calculated. The one-sample Student's t test with a Bonferroni correction was employed to assess the extent of facial asymmetry. Overall, for both genders, the facial features from the right side are larger than those from the left side. It was found that healthy Hispanic Caucasian children have fairly symmetric faces. However, the data suggest that there is a statistically significant asymmetry of facial features for healthy Hispanic Caucasian children. For example, the ratio of the nostril (sbal - sn) was asymmetric for healthy Hispanic Caucasian boys and the ratio between the Geodesic distances from tragion to nasion $(t-n)$ was asymmetric for both genders. The result of this study is consistent with previous studies of other racial/ethnic groups. This information will serve as reference data for surgeons who conduct reconstructive surgery for children with congenital deformities of their faces.
\end{abstract}

Keywords: reconstruction surgery, 3D stereophotogrammetry, facial asymmetry, facial features

\section{Introduction}

Congenital facial deformities can be detrimental to a child's self esteem, interpersonal relationship, and teacher student connections [1-3]. Current maxillofacial repair procedures rely on the artistic vision of the surgeon rather than a set of quantitative standards. An objective system for quantifying surgical outcomes is key for enabling the development of evidence-based approachs to reconstructive surgery.

In order to determine how the face of a patient should appear after reconstructive surgery, surgeons need precise knowledge of the craniofacial norms of the patient's race/ethnic group. Craniofacial norms of non-Hispanic Caucasians are well documented, while Hispanic Caucasians are less studied; yet, they comprise one of the largest and fastest growing ethnic groups in the U.S. The objective of this study was to conduct a quantitative analysis of facial asymmetry of healthy Hispanic Caucasian children residing in Central Texas, USA.

Previous studies [4-10] have consistently reported that there is a mild asymmetry of the face for different racial/ethnic groups. For example, Farkas and Cheung [4] reported a subtle asymmetry of 308 healthy, young North American Caucasians (154 boys and 154 girls). Primozic et al. [5] also showed a mild asymmetry of 27 healthy, young Slovenian Caucasians (15 boys and 12 girls) in their longitudinal study. Similar trends were found in other studies: 1) Ferrario et al. [6] of 80 healthy, young Caucasians (40 men and 40 women) residing in Italy, 2) White et al. [9] of 83 healthy Caucasian infants (41 boys and 42 girls) residing in the United Kingdom, and 3) Ercan et al. [7] of 321 healthy, young Turks (151 men and 170 women).

However, previous findings are variable with respect to the nature of the reported asymmetry. Some studies [4-6] reported that the right side of the face is larger that the left side, while it is not true for one study [7]. There was also variability in which facial part was noted as the most asymmetric ; some studies $[4,5]$ found that the upper part of the face is the most asymmetric part of the face, while other studies reported the middle [7] and the lower facial part [10] as the most asymmetric part of the face.

* mia.markey@utexas.edu; +1-512-471-1711; bmil.bme.utexas.edu 
This variation across the studies of different racial/ethnic groups suggests that we may need separate normative data for Hispanic Caucasian pediatric population.

In this study, we assessed the facial asymmetry of healthy Hispanic Caucasian children using three-dimensional (3D) surface images. Assessing the facial asymmetry using 3D surface image has advantages over the analysis on two-dimensional (2D) clinical photographs and the traditional direct anthropometry. 3D surface images preserve the depth information of the facial morphology, which is usually lost on $2 \mathrm{D}$ clinical photographs. Moreover, 3D surface images allow us to see a number of different views of the facial morphology, while $2 \mathrm{D}$ photographs only offer a limited number of views of the face. Although direct anthropometry on the study subject has been considered as the gold standard for evaluating the facial morphology, it requires substantial involvement of study subjects, which may burdensome to them. Once it is taken, which usually take only a few seconds, 3D facial morphology of study subjects can be evaluated anytime without bothering them. Moreover, many studies (e.g., [11, 12]) validated the usage of 3D surface imaging technique for evaluating the result of surgery.

\section{Materials and Methods}

\subsection{Dataset}

The dataset used for this study consists of 80 3D facial surface images of healthy Hispanic Caucasian children who are currently residing in Central Texas, USA. A total 41 boys and 39 girls whose ages ranged from 7 to 12 years were recruited under an approved IRB protocol. A 3dMDCranial system (3dMD, Atlanta, GA) was used to obtain all 3D facial surface images. Figure 1 shows an example 3D facial surface image of one study participant.

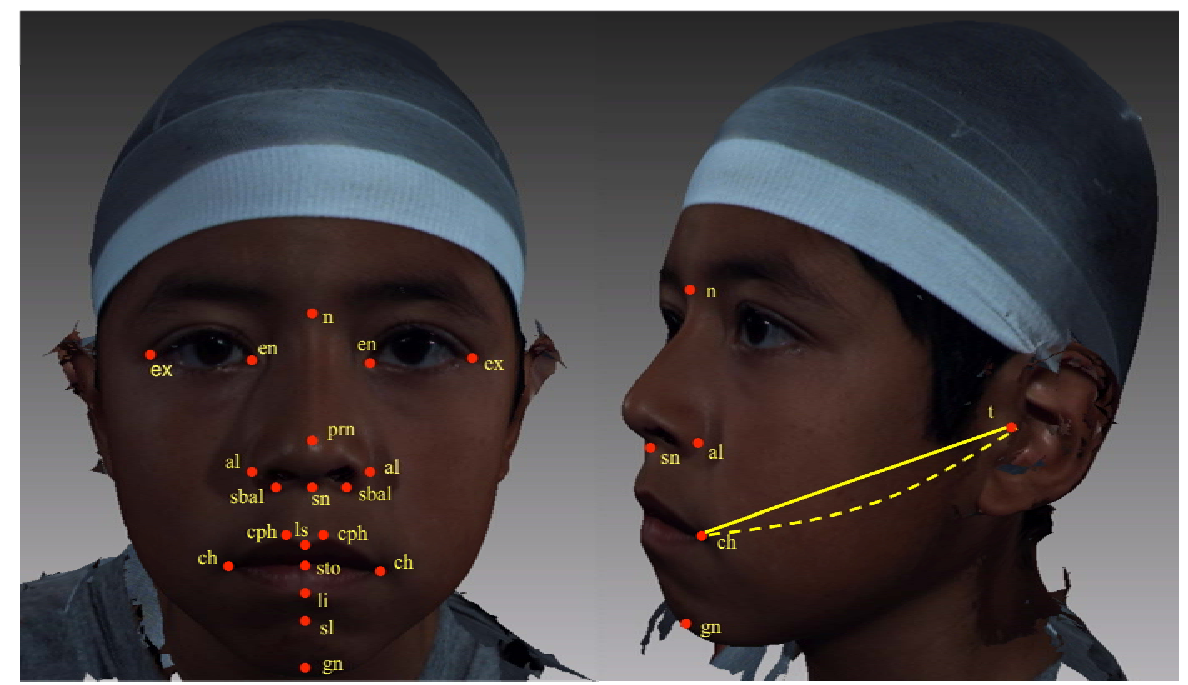

Fig. 1. Example 3D surface images for subjects enrolled for this study. Twenty-two fiducial points marked on a face. This figure illustrates an example of Euclidean (yellow solid line) and Geodesic (a.k.a., Surface distance, yellow dashed line) distances (right).

\subsection{Quantification of Morphology of Each Hemiface}

This study first quantified the morphology of each hemiface (left and right) of participants using 27 anthropometric distances (facial features) between 22 facial landmarks. These 22 facial landmarks of all study participants were annotated by one non-clinical observer (B.K.) following the definition based on [13]. Figure 1 summarizes the list of facial landmarks used for this study. The 27 anthropometric distances consist of two types of distances, which are simple Euclidean distances and Geodesic (Surface) distances. Dijkstra's shortest path algorithm [14] was used to compute the Geodesic distances. Table 1 shows the list of Euclidean and Geodesic distances used for this study and their corresponding summary statistics of the study participants. To access the facial asymmetry of healthy Hispanic Caucasian children, the ratio of each facial feature of the left hemiface to the corresponding facial feature of the right hemiface was calculated as follows,

$$
R_{i}=\frac{f_{i, \text { left }}}{f_{i, \text { right }}} \text { for all } i \text {, }
$$

where $R_{i}$ is the computed ratio for the $i^{\text {th }}$ facial feature obtained from left and right hemiface, which are $f_{i, \text { right }}$, and $f_{i, \text { efft }}$, respectively. 
Table 1. List of Euclidean and Geodesic distances used for this study and their corresponding summary statistics of study participants. (Unit: $\mathrm{mm}$ )

\begin{tabular}{|c|c|c|c|c|c|c|c|c|c|c|}
\hline \multirow{2}{*}{ Facial Area } & \multirow{2}{*}{ Distance Type } & \multirow{2}{*}{ List of Distances } & \multicolumn{2}{|c|}{ Boy Left } & \multicolumn{2}{|c|}{ Boy Right } & \multicolumn{2}{|c|}{ Girl Left $_{\text {L }}$} & \multicolumn{2}{|c|}{ Girl $_{\text {Right }}$} \\
\hline & & & Mean & SD & Mean & SD & Mean & SD & Mean & SD \\
\hline \multirow[t]{3}{*}{ Orbital } & Euclidean & ex-en & 30.3 & 2.4 & 30.3 & 2.9 & 22.7 & 29.9 & 29.3 & 3.6 \\
\hline & & en-n & 23.2 & 2.1 & 23.3 & 2.1 & 24.1 & 23.5 & 23.8 & 3.5 \\
\hline & Geodesic & en-n & 29 & 4.6 & 28.2 & 2.9 & 28.1 & 27.7 & 29.1 & 4.6 \\
\hline \multirow[t]{4}{*}{ Nasal } & Euclidean & al-prn & 22.6 & 3.4 & 23.3 & 3.7 & 20.3 & 23.5 & 23.8 & 4.1 \\
\hline & & sbal-sn & 11.5 & 2.4 & 12.5 & 2.6 & 7.8 & 11.4 & 12.1 & 2.1 \\
\hline & & al-sbal & 10 & 1.8 & 9.5 & 1.6 & 10.8 & 9.9 & 9.8 & 1.4 \\
\hline & Geodesic & al-prn & 28.6 & 5.4 & 28.3 & 5.1 & 24.7 & 28.1 & 28.7 & 5.5 \\
\hline \multirow[t]{9}{*}{ Oral } & Euclidean & cph-ls & 6.4 & 1.1 & 5.3 & 0.9 & 8.1 & 5.9 & 4.9 & 1.2 \\
\hline & & ch-ls & 28.8 & 2.2 & 28.7 & 2.5 & 25.3 & 28.4 & 28.2 & 2.7 \\
\hline & & ch-li & 25.4 & 2.6 & 26.3 & 2.7 & 24.1 & 25.6 & 25.9 & 2.7 \\
\hline & & ch-sto & 24.1 & 2.6 & 25 & 2.5 & 21.9 & 24.6 & 24.6 & 2.9 \\
\hline & & ch-sl & 26.7 & 2.4 & 28.2 & 2.5 & 27 & 26.9 & 27.9 & 2.5 \\
\hline & & ch-gn & 43 & 5.1 & 42.8 & 5 & 38.1 & 42 & 41.4 & 3.2 \\
\hline & & sbal-cph & 14.6 & 2.1 & 14.8 & 1.8 & 15 & 14.4 & 14.5 & 1.7 \\
\hline & & al-ch & 31.7 & 2.4 & 32 & 2.6 & 30.7 & 31 & 31.1 & 2.5 \\
\hline & Geodesic & N/A & & & & & & & & \\
\hline \multirow[t]{12}{*}{ Profile } & Euclidean & $t-s n$ & 115.2 & 6.6 & 116.4 & 6.6 & 119.3 & 113.8 & 114 & 6.3 \\
\hline & & t-gn & 126.4 & 8.7 & 127.9 & 9 & 125.1 & 124.9 & 124.7 & 7.4 \\
\hline & & $t-n$ & 111.3 & 4.9 & 112.8 & 5.2 & 116.8 & 111 & 111.8 & 6.1 \\
\hline & & t-ch & 98.3 & 7 & 99.8 & 7.2 & 101.9 & 96.7 & 97.2 & 5.8 \\
\hline & & $t-e x$ & 69.4 & 4.9 & 70.2 & 5.4 & 81.1 & 69.2 & 70.2 & 5.8 \\
\hline & & t-al & 100 & 6.5 & 100.5 & 6.1 & 107.8 & 98.6 & 98.5 & 6.5 \\
\hline & Geodesic & $t-s n$ & 146.1 & 9.9 & 148.7 & 9.6 & 156 & 146.9 & 146 & 10 \\
\hline & & $t-g n$ & 151.7 & 10.1 & 155.7 & 12.5 & 164.7 & 153 & 151.5 & 10.4 \\
\hline & & $t-n$ & 128.2 & 11.6 & 129.3 & 9.8 & 156 & 143.1 & 127.2 & 10.7 \\
\hline & & t-ch & 119.4 & 8.9 & 122.6 & 10.8 & 135.1 & 119.7 & 119.1 & 9 \\
\hline & & t-ex & 84.8 & 8.8 & 85.8 & 8.4 & 102.8 & 83.5 & 84.7 & 9 \\
\hline & & t-al & 127.6 & 10.7 & 129.3 & 9.8 & 142 & 127.4 & 127.2 & 10.7 \\
\hline
\end{tabular}

\subsection{Statistical Analysis}

As a perfectly symmetrical face is expected to have a ratio of 1 for all facial features, the one-sample Student's t test was employed to assess the extent of facial asymmetry. The null hypothesis for this study was that facial features are symmetric (i.e., the means of ratios of facial features are equal to 1 ). The test was performed on each gender group. A Bonferroni correction was employed as multiple statistical tests were conducted. The corrected significance level was 0.0019 . All analyses were performed using the MATLAB v.7.12.0 (R2011a) (The Mathworks, Natick, MA) statistics toolbox v.7.5 (R2011a).

\section{Results}

Table 2 shows the summary statistics of each facial feature and ratio, and the results of the statistical test for each gender group. Overall, for both genders, the facial features from the right side are larger than those from left side. We failed to reject the null hypothesis for more than half of the facial feature ratios (total 20 features for boys and total 23 features for girls), which indicates that healthy Hispanic Caucasian children have fairly symmetric faces. There was mild but not statistically significant ( $p$-value is between the cut-off statistical significance level before and after the Bonferroni correction) asymmetry found for both genders (total 3 features for boys and total 2 features for girls). For example, 
the ratio between left and right profile distances from cheilion to labiale inferius (ch - li) shows mild asymmetry but it is not statistically significant for boys. In addition, the ratio between left and right nostril (sbal - sn) shows mild asymmetry but it is not statistically significant for girls.

Table 2. Summary statistics and statistical test results for each ratio.

\begin{tabular}{|c|c|c|c|c|c|c|c|c|}
\hline \multirow{2}{*}{ Facial Area } & \multirow{2}{*}{ Distance Type } & \multirow{2}{*}{ List of distances } & \multicolumn{3}{|c|}{ Ratio Boy } & \multicolumn{3}{|c|}{ Ratio $_{\text {Girl }}$} \\
\hline & & & Mean & SD & $p$-value & Mean & SD & $p$-value \\
\hline \multirow[t]{3}{*}{ Orbital } & Euclidean & ex-en & 1.01 & 0.1 & 0.6831 & 1.03 & 0.12 & 0.1439 \\
\hline & & en-n & 1 & 0.11 & 0.8734 & 1 & 0.13 & 0.9499 \\
\hline & Geodesic & en-n & 0.99 & 0.13 & 0.558 & 0.97 & 0.14 & 0.1496 \\
\hline \multirow[t]{4}{*}{ Nasal } & Euclidean & al-prn & 0.98 & 0.12 & 0.2427 & 0.99 & 0.12 & 0.6965 \\
\hline & & sbal-sn & 0.92 & 0.13 & $0.0005^{\star}$ & 0.94 & 0.14 & $0.019^{* *}$ \\
\hline & & al-sbal & 1.07 & 0.19 & $0.0245^{\star *}$ & 1.02 & 0.16 & 0.5503 \\
\hline & Geodesic & al-prn & 0.97 & 0.16 & 0.2019 & 0.99 & 0.15 & 0.6789 \\
\hline \multirow[t]{9}{*}{ Oral } & Euclidean & cph-Is & 1.24 & 0.29 & $<0.0001^{*}$ & 1.29 & 0.48 & $0.0004^{*}$ \\
\hline & & ch-ls & 1.01 & 0.07 & 0.5156 & 1.01 & 0.09 & 0.3594 \\
\hline & & ch-li & 0.97 & 0.08 & $0.0137^{\star *}$ & 0.99 & 0.09 & 0.6174 \\
\hline & & ch-sto & 0.97 & 0.1 & 0.0528 & 1.01 & 0.13 & 0.7542 \\
\hline & & ch-sl & 0.95 & 0.07 & $<0.0001^{*}$ & 0.97 & 0.07 & $0.0053^{* *}$ \\
\hline & & ch-gn & 1 & 0.04 & 0.47 & 1.01 & 0.04 & 0.0291 \\
\hline & & sbal-cph & 0.98 & 0.08 & 0.1904 & 0.99 & 0.1 & 0.7494 \\
\hline & & al-ch & 0.99 & 0.06 & 0.3477 & 1 & 0.07 & 0.9225 \\
\hline & Geodesic & N/A & & & & & & \\
\hline \multirow[t]{12}{*}{ Profile } & Euclidean & $t-s n$ & 0.99 & 0.04 & 0.1673 & 1 & 0.04 & 0.9529 \\
\hline & & $t-g n$ & 0.99 & 0.03 & 0.0512 & 1 & 0.04 & 0.7927 \\
\hline & & $t-n$ & 0.99 & 0.04 & $0.0324^{* *}$ & 0.99 & 0.04 & 0.3457 \\
\hline & & $\mathrm{t}$-ch & 0.99 & 0.05 & 0.0839 & 1 & 0.05 & 0.6208 \\
\hline & & t-ex & 0.99 & 0.07 & 0.4853 & 0.99 & 0.08 & 0.3944 \\
\hline & & $\mathrm{t}$-al & 1 & 0.05 & 0.7006 & 1 & 0.05 & 0.8396 \\
\hline & Geodesic & $t-s n$ & 1 & 0.06 & 0.6844 & 1.01 & 0.06 & 0.3972 \\
\hline & & $t-g n$ & 1 & 0.05 & 0.5155 & 1.01 & 0.05 & 0.2155 \\
\hline & & $t-n$ & 1.12 & 0.07 & $<0.0001^{*}$ & 1.13 & 0.07 & $<0.0001^{*}$ \\
\hline & & $\mathrm{t}-\mathrm{ch}$ & 1 & 0.07 & 0.9103 & 1.01 & 0.07 & 0.5494 \\
\hline & & t-ex & 0.98 & 0.09 & 0.0957 & 0.99 & 0.1 & 0.5683 \\
\hline & & t-al & 1 & 0.06 & 0.8303 & 1 & 0.07 & 0.7195 \\
\hline
\end{tabular}

However, our data suggest that there is statistically significant asymmetry of some facial features for healthy Hispanic Caucasian children (total 4 features for boys and total 2 features for girls). For healthy boys, the ratios between left and right Euclidean distances between: 1) subalare (sbal) and subnasale (sn), 2) crista philtr (cph) and labiale superius (Is), 3) cheilion (ch) and sublabiale (sl) show statistically significant asymmetry. In addition, the ratios between left and right Geodesic distances between tragion $(t)$ and nasion $(n)$ shows statistically significant asymmetry. Similar trends were found for healthy girls. For this gender group, Euclidean distance ratios between crista philtr (cph) and labiale superius (Is) and Geodesic distance ratio between tragion ( $\mathrm{t}$ ) and nasion ( $\mathrm{n}$ ) show statistically significant asymmetry. Figure 2 illustrates the facial features showing statistically significant asymmetry. 


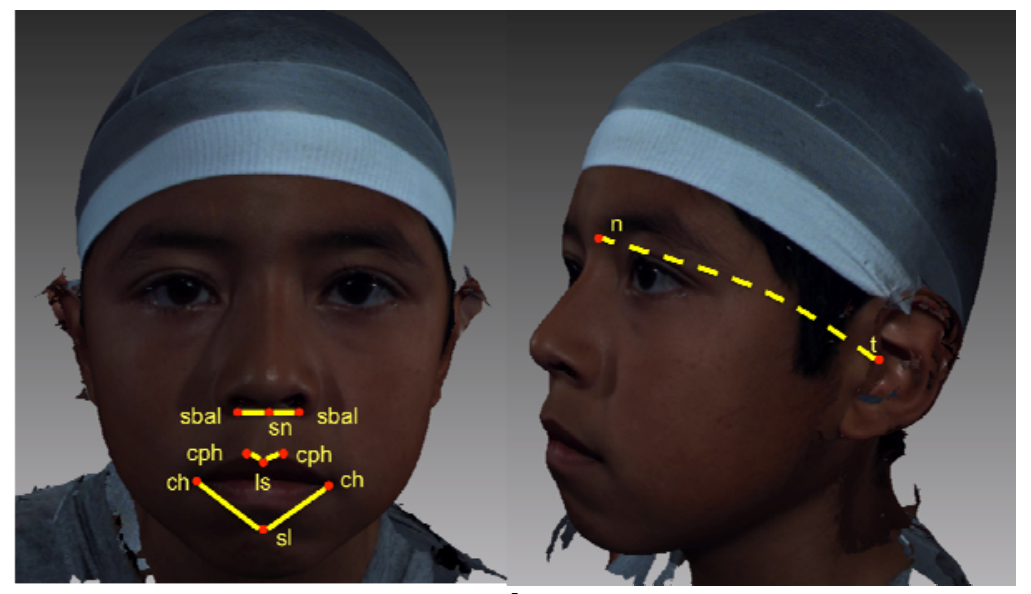

A

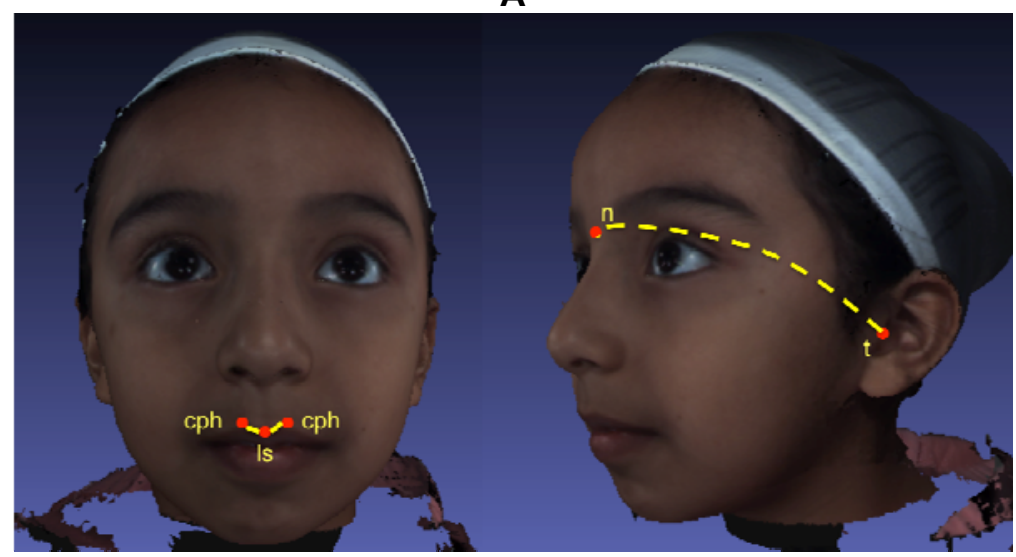

B

Fig. 2. Graphical illustration of facial features that show statistically significant asymmetry for boy (A) and girl (B). The yellow solid line and yellow dashed line represent Euclidean and Geodesic distances, respectively.

\section{Discussion}

In this study, we assessed the facial asymmetry of healthy Hispanic Caucasian children. We considered two types of distances, which are Euclidean and Geodesic (surface) distances, between manually annotated facial landmarks. The ratio of those distances (or facial features) from left and right hemiface were used to seek possible asymmetry on the facial morphology of the study population.

We found in general that the facial features from the right side are larger than those from left side for both genders. This is consistent with the aforementioned studies of other racial/ethnic groups conducted by Farkas and Cheung [4] (North American Caucasians), Primozic et al. [5] (Slovenian Caucasians), and Ferrario et al. [6] (Caucasians in Italy); however, this result is contrary of the finding of Ercan et al.'s analysis of a young Turkish sample [7]. Interestingly, for both genders, the ratio of Euclidean distances from crista philtr (cph) to labiale superius (Is) and Geodesic distances from tragion $(t)$ and nasion $(n)$ show that the left side is statistically significantly wider than the right side, which is opposite of general finding of this study. Although we cannot make a direct comparison due to the difference on the age (7-12 years old vs. 18 years old) and the distance metric (Geodesic vs. projective), the oldest group of study subjects in Farkas and Cheung [4] showed a similar asymmetry on the projective distance made from tragion $(\mathrm{t})$ to nasion $(\mathrm{n})$ by a spreading caliper. However, there is no consistent finding for the aged match group in their study to our data. A similar trend was found in the study of Ercan et al. [7] for the cph-ls. Although we cannot make a direct comparison due to the difference on the age (7-12 years vs. 17-21 years), they reported a similar result of ours in that the left side of cph-ls is wider than the right side for male subjects.

The comparisons from the data of this study to those of others show that there is a distinct trend on the facial morphology of healthy Hispanic Caucasian children residing central Texas, USA. Although the study population has fairly symmetrical face, there exist unique asymmetries different from other racial/ethnic groups. This information may serve as reference data for both plastic surgeons and 
orthodontists who conduct reconstructive surgery for children with congenital deformities of their faces. Reconstructive surgery of patients with facial asymmetry provides a challenge. When severity increases and is complex, it is hard to judge and plan for surgical correction. Often times, the asymmetry affects the "normal side" and patients may prefer the affected side as opposed to the non-affected side. Knowing that there are inherited asymmetries in certain areas of the face present in either boys or girls should be taken in consideration prior to any surgical planning.

Additional applications of these normative data are constructing soft tissue norms for surgical software and prediction of changes of soft tissue with surgery. Moreover, normative data could be used for construction of better fitting oxygen masks and CPAP masks for children. Ultimately, understanding the facial form of Hispanic Caucasian children will enable the development of better treatment tools in the future for this large growing segment of our population.

\section{References}

1. Kagian, A., et al., (2008): "A machine learning predictor of facial attractiveness revealing human-like psychophysical biases", in Vision Research. p. 235-243.

2. Shaw, W.C., (1981): "The influence of children's dentofacial appearance on their social attractiveness as judged by peers and lay adults.", Am J Orthod, 79(4): " p. 399-415.

3. Shaw, W.C. and S. Humphreys, (1982): "Influence of children's dentofacial appearance on teacher expectations.", Community Dent Oral Epidemiol, 10(6): p. 313-9.

4. Farkas, L.G. and G. Cheung, (1981): "Facial asymmetry in healthy North American Caucasians.", An anthropometrical study. The Angle Orthodontist, 51(1): p. 70-77.

5. Primozic, J., et al., (2012): "Assessment of facial asymmetry in growing subjects with a three-dimensional laser scanning system.", Orthodontics \& Craniofacial Research.

6. Ferrario, V.F., et al., (1994): "Distance from symmetry: a three-dimensional evaluation of facial asymmetry.", Journal Of Oral And Maxillofacial Surgery: Official Journal Of The American Association Of Oral And Maxillofacial Surgeons, 52(11): p. 1126-1132.

7. Ercan, I., et al., (2008): "Facial asymmetry in young healthy subjects evaluated by statistical shape analysis.", Journal Of Anatomy, 213(6): p. 663-669.

8. Toma, A.M., et al., (2008): "A three-dimensional look for facial differences between males and females in a British-Caucasian sample aged 15 1/2 years old.", Orthodontics \& Craniofacial Research, 11(3): p. 180-185.

9. White, J.E., et al., (2004): "Three-dimensional facial characteristics of Caucasian infants without cleft and correlation with body measurements.", The Cleft Palate-Craniofacial Journal: Official Publication Of The American Cleft Palate-Craniofacial Association, 41(6): p. 593-602.

10. Severt, T.R. and W.R. Proffit, (1997): "The prevalence of facial asymmetry in the dentofacial deformities population at the University of North Carolina.", The International journal of adult orthodontics and orthognathic surgery, 12(3): p. 171-176.

11. Weinberg, S.M., et al., (2004): "Digital three-dimensional photogrammetry: evaluation of anthropometric precision and accuracy using a Genex 3D camera system.", The Cleft Palate-Craniofacial Journal: Official Publication Of The American Cleft Palate-Craniofacial Association, 41 (5 (Print)): p. 507-518.

12. Weinberg, S.M., et al., (2006): "Anthropometric precision and accuracy of digital three-dimensional photogrammetry: comparing the Genex and 3dMD imaging systems with one another and with direct anthropometry.", The Journal Of Craniofacial Surgery, 17(3 (Print)): p. 477-483.

13. Farkas, L.G., (1994): "Anthropometry of the Head and Face.", Second Edition ed., New York: Raven Press.

14. Dijkstra, E.W., (1959): "A note on two problems in connexion with graphs.", Numerische Mathematik, 1(1): p. 269-271. 